\title{
Xihong Hu, Guoying Huang, Mier Pa. Multidetector Computed Tomography for Assessing a Pulmonary Artery Sling in a Pediatric Patient. Pediatr Cardiol 2008; 29:1006-1007
}

\author{
George C. Kagadis · Eugenia C. Panagiotopoulou • \\ Kostas N. Priftis · Michael B. Anthracopoulos
}

Received: 9 December 2008/ Accepted: 2 February 2009/Published online: 18 March 2009

(C) Springer Science+Business Media, LLC 2009

To the Editor,

We read with interest the case report by $\mathrm{Hu}$ et al.[1] that described the use of multidetector computed tomography angiography (MDCTA) in defining the anatomy of a pulmonary artery sling in a 4-month-old infant. By presenting both axial and maximum intensity projection images, the author clearly demonstrated the merits of multiplanar reformatting and of techniques that produce three-dimensional (3-D) images in providing a "userfriendly" representation of the anatomy of airway lesions in children, which can greatly assist accurate diagnosis and preoperative planning.

We recently reported, for the first time in the pediatric literature, on the preoperative evaluation of the trachea in a 4-year-old female with pulmonary artery sling accompanied by long-segment tracheal stenosis owing to complete cartilaginous rings ("ring-sling complex," "stove-pipe trachea," "funnel-like," or "carrot-like stenosis") using external 3-D CT and virtual bronchoscopy (VB) images obtained by a volume-rendering technique that were compared to fiberoptic bronchoscopy (FOB); in our report we also included a comprehensive review of the use of VB in pediatric patients in the English literature [2]. Many

\section{G. C. Kagadis}

Department of Medical Physics, School of Medicine,

University of Patras, Rio 26504, Greece

E. C. Panagiotopoulou · M. B. Anthracopoulos ( $\varangle)$

Respiratory Unit, Department of Pediatrics, School of Medicine,

University of Patras, Rio 26504, Greece

e-mail: manthra@otenet.gr

\section{K. N. Priftis}

Department of Allergology-Pulmonology,

Penteli Children's Hospital, Athens 15236, Greece surgeons require detailed preoperative information on the anatomy of the tracheobronchial tree [3]. Although dynamic changes of the airways during respiration and external pulsation of an adjacent artery (vascular ring) can only be assessed by FOB, VB helped us "bypass" the stenotic segment of the tracheal lumen (this was not possible with the 3.6-mm-diameter fiberoptic instrument) and demonstrate normal-sized orifices of the main bronchi. Three-dimensional CT and VB also enabled us to accurately assess the length and degree of tracheal stenosis in planning the surgical repair of the malformation.

\section{References}

1. Hu X, Huang G, Pa M (2008) Multidetector computed tomography for assessing a pulmonary artery sling in a pediatric patient. Pediatr Cardiol 29:1006-1007

2. Kagadis GC, Panagiotopoulou EC, Priftis KN et al (2007) Preoperative evaluation of the trachea in a child with pulmonary artery sling using 3-dimentional computed tomographic imaging and virtual bronchoscopy. J Pediatr Surg 42:E9-E13

3. Valletta EA, Pregarz M, Bergamo-Andreis IA, Boner AL (1997) Tracheoesophageal compression due to congenital vascular anomalies (vascular rings). Pediatr Pulmonol 24:93-105 